\title{
Introduction : pour une analyse rhétorique des textes politiques
}

La « microlecture » de Michael Leff au prisme des traditions analytiques françaises

Introduction: Rhetorical Criticism and Public Address. Michael Leff's Close

Reading and French Contemporary Research

\section{Ruth Amossy}

\section{(2) OpenEdition}

12 Journals

Édition électronique

URL : http://journals.openedition.org/aad/1081

DOI : 10.4000/aad.1081

ISSN : 1565-8961

Éditeur

Université de Tel-Aviv

Référence électronique

Ruth Amossy, "Introduction: pour une analyse rhétorique des textes politiques », Argumentation et Analyse du Discours [En ligne], 6 | 2011, mis en ligne le 15 avril 2011, consulté le 23 septembre 2019 URL : http://journals.openedition.org/aad/1081 ; DOI : 10.4000/aad.1081

Ce document a été généré automatiquement le 23 septembre 2019.

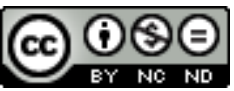

Argumentation \& analyse du discours est mis à disposition selon les termes de la licence Creative Commons Attribution - Pas d'Utilisation Commerciale - Pas de Modification 4.0 International. 


\title{
Introduction : pour une analyse rhétorique des textes politiques
}

La « microlecture » de Michael Leff au prisme des traditions analytiques françaises

\author{
Introduction: Rhetorical Criticism and Public Address. Michael Leff's Close \\ Reading and French Contemporary Research
}

\section{Ruth Amossy}

1 La rhétorique américaine n'est guère connue dans les pays francophones, et on peut s'interroger sur les raisons de ce cloisonnement à une époque dite de «mondialisation». Il y a, sans doute, l'obstacle des langues (bien qu'en l'occurrence, l'anglais soit désormais lu par la majorité des chercheurs francophones). Mais il y a, surtout, le poids des traditions et le découpage variable des disciplines. La rhétorique aux Etats-Unis dont parle David Zarefsky dans le présent numéro s'est développée dans un contexte sociopolitique et dans des cadres d'enseignement qui diffèrent profondément de ceux qu'on trouve en France. Contrairement à ce qui se passe dans l'hexagone et dans les pays de langue française, la rhétorique est en bonne place dans l'université américaine. En plus des départements de rhétorique, l'enseignement de la discipline s'y pratique dans les deux domaines qui s'en revendiquent haut et fort: English and Composition (Anglais et Composition), et Speech Communication (aujourd'hui plutôt «Communication» tout court). L'enseignement théorique s'y accompagne souvent d'une pratique de l'art oratoire, du débat et de l'écriture argumentée. Il suffit par ailleurs de voir l'affluence des chercheurs qui viennent aux Congrès biannuels de la RSA (Rhetorical Society of America) pour se persuader de l'enracinement de la rhétorique dans les cursus américains.

2 Cet ancrage institutionnel a donné naissance à une réflexion théorique en constante ébullition et à une impressionnante floraison d'études sur l'art oratoire américain, mais aussi sur de nombreuses autres formes de discours. Elle a engendré une série de revues savantes dont on trouvera le détail dans la «Bibliographie sélective annotée des publications en langue anglaise sur la rhétorique, la critique rhétorique et 
l'argumentation" que Michael Leff avait élaborée avec Steven R. Edscorn pour le numéro 2 d'Argumentation et Analyse du Discours (2009). En plus des références aux collections consacrées à la rhétorique et aux ouvrages qui retracent l'histoire de la discipline aux Etats-Unis, on y trouvera également une liste de livres et articles sélectionnés dans une masse impressionnante de travaux touchant au discours politique, aux mouvements sociaux, à la religion, aux sciences, à la littérature, etc. La rhétorique aux Etats-Unis a ainsi sa propre histoire, qui est déjà longue (l'étude de ce qui a été dénommé Public Address a commencé il n'y pas moins de 75 ans). On voit que la situation américaine diffère profondément de ce qu'on peut trouver dans les pays francophones où la rhétorique n'est pas, de nos jours, une discipline institutionnalisée même si elle se glisse dans certains cursus (comme ceux de stylistique, par exemple) et compte d'illustres spécialistes de rhétorique antique ou d'histoire de la rhétorique comme Marc Fumaroli, voire de remarquables théoriciens comme Michel Meyer en Belgique ou Marc Angenot au Québec.

3 Michael Leff était profondément engagé dans tous les débats qui ont accompagné le développement des études de rhétorique aux Etats-Unis et, dans ses articles, il prend soin de se positionner par rapport aux options qu'offraient au même moment des courants concurrents. Ses travaux suivent ainsi l'évolution de la discipline; ses écrits portent la marque de ses questionnements et s'inscrivent dans ses controverses. Ce faisant, il développe et affine cependant au fil du temps une approche originale de l'étude rhétorique des textes politiques qui appelle notre attention par sa fécondité et par le mélange de similitudes et de différences qu'elle présente par rapport aux démarches qui nous sont familières ${ }^{1}$.

4 Leff était au départ, comme en atteste la plupart de ses écrits, un spécialiste de l'Antiquité féru d'Aristote, mais aussi de Cicéron, et attentif aux questions qui se trouvent au centre de l'art oratoire comme le rapport au public, les topiques ou l'ethos. C'est, paradoxalement, par un retour aux sources qu'il a voulu dépasser les aspects surannés des démarches qualifiées de son temps de néo-aristotélisme (dont il traite dans « Des mots au plus près des choses", traduit dans ce numéro). Pour redonner ses lettres de noblesse à l'étude du discours public dans la cité, une branche de savoir dont l'importance en régime démocratique ne peut faire aucun doute, il a proposé un retour aux textes considérés comme une pratique oratoire: une parole dirigée vers un auditoire concret, une performance qui s'adapte à des circonstances précises pour faire adhérer le public à une thèse. S'élevant contre l'application mécanique de catégories préexistantes qui fragmente le texte et en laisse échapper l'essentiel, une voie souvent adoptée par les néo-aristotéliciens, Leff se fonde sur Cicéron pour saisir le texte oratoire, en l'occurrence le discours politique, comme une pratique qu'il faut appréhender dans son mouvement et sa cohérence propres. A l'analyste incombe donc la tâche d'en dévoiler le fonctionnement discursif et la dynamique persuasive. Contrairement à ceux qui se tournent vers des unités plus larges - regroupements divers de textes, formations discursives, ensembles idéologiques - Leff voit dans la performance singulière le cœur de l'art oratoire, et privilégie l'analyse approfondie d'un texte politique particulier qu'il sélectionne souvent, en harmonie avec la tradition, parmi les grands discours de l'Histoire américaine. Ses études du discours de Lincoln à Cooper Union ou de la lettre de prison de Martin Luther King, dans le présent numéro, en témoignent. 
5 C'est dans cette perspective que Leff, se situant dans l'espace global de la «critique rhétorique » américaine, se réclame du close reading, une approche dont le nom n'a pas d'équivalent entériné en français et qui consiste dans la lecture minutieuse d'un discours singulier. Notons que Leff a aussi utilisé textual criticism, critique textuelle (1992), pour souligner qu'il mettait l'accent sur le texte. Nous utiliserons ici le terme de «microlecture", appellation qui, malgré les risques d'association avec l'ouvrage essentiellement thématique du même nom de Jean-Pierre Richard, nous semble plus évocateur que "commentaire de texte " et même "analyse textuelle " (mais nous laissons entre parenthèses l'expression anglaise de close reading dans les traductions). Il ne s'agit pas, dans cette microlecture, de s'attacher à des détails minimes ou de privilégier des fragments, mais d'effectuer une analyse serrée, en quelque sorte à ras de texte, d'un discours oratoire singulier.

6 C'est donc le fonctionnement discursif d'une entité textuelle close qu'il s'agit d'explorer en entrant dans l'épaisseur du texte oral ou écrit. De ce point de vue, la critique rhétorique soumet des textes appartenant au discours public et politique comme l'allocution présidentielle, le discours de meeting, les débats électoraux, la lettre ouverte, à une analyse que la tradition américaine voulait réserver au texte littéraire (voir à ce propos l'introduction de Zarefsky). A l'instar de l'analyse du discours instiguée par Dominique Maingueneau en France (2004), Leff refuse de dresser des barrières étanches entre le littéraire et le non-littéraire, et plaide pour la nécessité de dégager la particularité et la complexité des discours politiques au même titre que celles des œuvres poétiques et fictionnelles.

7 Et en effet, dans la mesure où, pour les tenants du close reading, les discours politiques ont leur cohérence et leur dynamique interne, ils présentent une véritable texture - un entrecroisement de fils qui tissent une configuration singulière (Jasinski 2001 : 92). La métaphore de Leff qui emploie souvent le terme de thick reading - une lecture qui entre dans l'épaisseur des textes - est ici parlante. S'ils ne se confondent pas avec le texte littéraire dans la mesure où celui-ci ne se veut pas une action directe sur le monde, les discours politiques n'en bénéficient pas moins d'un statut qui appelle à une investigation rigoureuse de ce qui se déploie à leur surface verbale. Il ne s'agit donc pas de les traverser pour en dégager les contenus ou en résumer les thèmes : une attention soutenue à leur structure et leur texture est de règle.

On peut dès lors parler - même si Leff n'a guère recours aux procédures et aux notions des sciences du langage dont son travail ne se réclame $\mathrm{pas}^{2}$ - d'une tentative d'explorer le fonctionnement verbal de l'allocution publique. Le rhétoricien adopte une démarche analytique qui entend dévoiler, dans le texte, how it works - comment ça marche, comment ça fonctionne. Pour ce faire, il lui faut prendre en compte la spécificité du discours politique qui, contrairement au texte poétique, n'a pas vocation à attirer l'attention sur le travail formel qui l'engendre : il a bien plutôt tendance à le camoufler, à le cacher en en effaçant les traces (Leff 1986: 381). C'est donc à l'analyste de les retrouver et de les mettre au jour.

9 Leff adopte le point de vue pragmatique qui voit dans la parole un acte à valeur sociale et, s'il propose d'entrer dans l'épaisseur du texte et d'en saisir le mouvement interne, c'est pour voir comment il cherche à réaliser par le pouvoir du verbe sa visée d'influence sociale. Dans sa pratique comme dans les réflexions qu'il mène sur la microlecture (close reading), il prend soin de mettre en garde contre les dangers d'une analyse purement interne: «La signification rhétorique, bien évidemment, n'a rien 
d'autotélique; elle a pour vocation d'atteindre le monde extérieur situé au-delà du texte, et de guider la compréhension et le comportement de l'auditoire dans cet univers » (Leff 1990, traduit dans le présent numéro, paragraphe 9). Cette orientation fermement établie veille à distinguer clairement les tendances formalistes que le New Criticism avait mises à l'ordre du jour dans les études littéraires, de la microlecture des discours politiques qui refuse de se laisser fasciner par les formes et les structures internes du texte. Tourné vers le monde extérieur sur lequel il veut laisser son empreinte, le discours public (public address) sous ses diverses formes est nécessairement ancré dans une situation précise. Connaître à fond les circonstances sociohistoriques et les intégrer pleinement à l'analyse formelle est indispensable pour saisir la visée et le sens d'un texte donné. En termes d'analyse du discours, l'analyse doit prendre en compte la situation de discours dans laquelle la parole nouvelle se déploie. Lire de près une allocution présidentielle ou une lettre ouverte, c'est articuler les formes discursives et les lieux socio-institutionnels dans lesquelles elles s'élaborent à un moment historique précis ${ }^{3}$.

10 «Dans cette perspective, l'analyse du discours a affaire de manière privilégiée avec les genres de discours", précise le Dictionnaire d'Analyse du Discours de Maingueneau et Charaudeau (2002: 48). Pour lier une perspective formelle attentive au travail du texte à ses conditions de production et de réception, Leff propose lui aussi de recourir aux genres qui contraignent la parole de l'orateur et en même temps lui ouvrent ses possibilités. C'est sur ce point que Leff et Mohrman insistent dans leur double étude de l'allocution de Lincoln à Cooper Union où, après une analyse serrée du texte, ils se penchent sur les principes qui ont guidé cette même lecture. L'analyse formelle ne doit ni se fragmenter en un relevé purement mécanique des procédés qui parsèment le texte, comme le faisait trop souvent l'analyse néo-aristotélicienne, ni rester aux alentours du texte en demeurant prise dans le détail historique et biographique. Il s'agit de retrouver la dynamique qui assure la cohérence de l'allocution et celle-ci, si elle est toujours singulière, n'en est pas moins en prise sur le genre dont elle relève. Ainsi, dans le discours de Lincoln à Cooper Union, Leff et Mohrman montrent comment l'indexation au genre du discours électoral explique la logique d'une démarche sur laquelle on s'est souvent mépris. Fidèle aux règles du discours de campagne, Lincoln se positionne par rapport aux autres candidats républicains et construit un ethos rhétorique de présidentiable à un moment où les élections proches se jouaient largement sur la question violemment controversée de la législation sur l'esclavage. Une analyse minutieuse des divers mouvements du texte permet de montrer comment ils se rapportent à cette visée centrale et d'écarter les interprétations qui ont accusé Lincoln de laxisme envers le Sud, en soulignant bien plutôt l'habilité politique d'un art oratoire où se construit de toutes pièces une figure de dirigeant républicain fiable et de futur président. Le recours de l'analyste au modèle générique, c'est-à-dire à une forme globale instituée, fait l'objet d'une réflexion approfondie dans l'article qui suit immédiatement l'analyse pour la situer dans la théorie aristotélicienne des genres (voir ici-même, « Lincoln à Cooper Union : Plaidoyer pour une critique néo-classique »).

11 Il est intéressant de voir comment Leff négocie son allégeance à la théorie des genres rhétoriques. En effet, il se réfère prioritairement à Aristote et à sa fameuse tripartition entre le délibératif, le juridique et l'épidictique fondée sur une perspective abstraite qui permet de regrouper en classes les discours individuels et de prendre en compte leurs caractères formels communs. Mais quand il en vient à l'allocution de Lincoln, il reconnaît que le discours électoral relève à la fois du délibératif (qui examine une 
politique en traitant de l'utile et du nuisible) et de l'épidictique (qui appelle au blâme ou à la louange d'un individu). Aucun des genres traditionnels n'étant satisfaisant pour rendre compte du discours de l'homme politique américain, il incombe à l'analyste de décrire les nombreux genres contemporains dont l'Antiquité ne peut bien évidemment rendre compte. L'ancrage dans le classicisme n'empêche pas Leff d'actualiser son étude et d'appeler à la description des genres multiples, à la fois oraux et écrits, dont se nourrit le discours politique moderne et contemporain. Même si Leff dit s'en tenir à la théorie aristotélicienne, on retrouve par ce biais la notion de "genres de discours " chère à l'analyse du discours, qui déborde largement la notion des genres rhétoriques dans leur version classique et permet de se livrer à une investigation pertinente des formes multiples du discours politique contemporain.

12 A cela s'ajoute la centralité de l'auditoire visé par le texte, et une étude attentive des modalités selon lesquelles le discours politique tient compte des particularités de ce public et s'adapte à lui. Comme l'argumentation dans le discours (Amossy 2010 [2000]) et une grande partie des travaux de langue française axés sur l'argumentation ou la prenant en compte, Leff met au centre de sa réflexion l'axe orateur-auditoire en s'appuyant sur la rhétorique classique, mais aussi sur la nouvelle rhétorique de Perelman et Olbrechts-Tyteca (1958). En même temps, il insiste dans «Perelman, argument ad hominem et ethos rhétorique " (paru en français dans Argumentation et Analyse du Discours 2009: paragraphe 24), sur le fait que Perelman n'étudie pas les modalités concrètes de la communication avec l'auditoire, si bien que « l'ouvrage relève et explicite méthodiquement les structures et les techniques argumentatives, et ce de façon très productive, mais ne fournit pas de descriptions en profondeur de la pratique rhétorique». Or, c'est dans cette pratique que se joue pour Leff la relation entre l'orateur et son auditoire. Ainsi, dans ses travaux sur la lettre de prison de Martin Luther King, il étudie d'abord le texte en fonction de l'auditoire blanc modéré qui en était le public-cible (cette analyse figure dans le présent numéro); mais dans une étude ultérieure de 2004, il précise que la lettre ouverte de King était également appelée à circuler parmi les Afro-Américains, dont la participation à l'action non-violente était essentielle pour la réussite de l'entreprise de King. Dans cette perspective, le texte, en construisant l'ethos de l'orateur, propose au lecteur noir un modèle d'activisme mais aussi de contrôle de soi qui doit lui permettre de devenir un acteur sur la scène politique et de la transformer en profondeur sur la base des valeurs communes de la nation. L'analyse explore ainsi concrètement la façon dont le texte se construit sur une double portée pour s'adresser simultanément à deux auditoires distincts.

Attention portée au fonctionnement discursif des discours institués et plus particulièrement du discours politique, prise en compte de la situation de discours, articulation d'une organisation formelle et d'un lieu sociopolitique, place centrale accordée à l'interrelation locuteur/allocutaire(s) et aux genres de discours - ce résumé délibérément formulé en termes d'analyse du discours vise à souligner les points communs entre ce courant français et l'approche du rhétoricien américain. Il faut ajouter que dans l'accent mis sur la tentative verbale de faire adhérer l'autre à un point de vue, il se rapproche forcément de l'argumentation dans le discours, à la fois dans le sens précis que lui donne Amossy (2010 [2000]) et dans le sens large de tous ceux qui entendent intégrer à l'analyse du discours l'étude de la visée d'influence sociale du discours (Charaudeau 2009). 
14 Aussi n'est-ce pas par hasard qu'une rencontre se produit entre les chercheurs de langue française et le rhétoricien américain sur la question de l'ethos et de l'argument ad hominem. Sans doute, Leff reprend la notion d'ethos rhétorique non comme l'image que tout discours construit ipso facto de son locuteur (conception courante de l'analyse du discours et de l'argumentation dans le discours (Maingueneau 2004; Amossy 2010b), mais comme moyen de preuve au sens d'Aristote. Cependant, il en réalise une étude discursive et argumentative fine en montrant comment l'image de soi se construit dans le texte, par quels moyens verbaux et en vue de quels enjeux sociopolitiques. Cette étude est au cœur de son analyse de la parole électorale de Lincoln, mais aussi de celle des débats entre Bush et Kerry, ou encore du texte de Martin Luther King dont Leff montre qu'il construit une image d'énergie contrôlée, de personne active et engagée, mais en même temps raisonnable et posée, susceptible d'assurer sa crédibilité en tant que dirigeant du mouvement d'action non-violente contre la ségrégation raciale.

En même temps, et à l'instar de l'argumentation dans le discours, Leff se préoccupe d'articuler l'analyse rhétorique et les théories de l'argumentation, dont il suit les développements et dont il se distancie néanmoins en raison du souci de validité logique propre à une approche théorique et normative coupée de l'usage effectif des arguments. En place d'une argumentation désincarnée, la critique rhétorique fondée sur la microlecture examine des cas particuliers non pour établir des critères généraux de validité, mais pour évaluer la façon dont un argument donné, voire un paralogisme, est utilisé dans une situation de communication précise en vue de provoquer certains effets. Comme Ruth Amossy dans son travail sur l'ad hominem (2002), Leff se réfère à Brinton (1985) pour montrer que l'attaque sur la personne tire sa pertinence de l'importance accordée à l'ethos ou crédibilité de l'orateur, et que celui-ci construit son image de soi en rapport et en opposition avec celle dont il affuble son adversaire. Dans cette perspective, il met en lumière les fonctions constructives de l'ad hominem dans les discours concrets qui l'exploitent à leur profit. Leff en offre une démonstration poussée dans sa réplique à Trudy Govier (représentante de la logique informelle) au sein de l'étude déjà mentionnée sur King où il montre comment le dirigeant noir construit un ethos qui répond aux accusations personnelles d'outsider et d'agitateur lancées contre lui ; mais il en traite aussi dans son texte sur Perelman (Argumentation et Analyse du Discours 2009 : 2), ainsi que dans son analyse des débats entre Bush et Kerry au moment des Présidentielles américaines de 2004 (traduit dans ce numéro). Dans cette étude de la polémique qui a opposé les deux candidats, Leff montre comment Bush, sous ses dehors d'orateur fruste au parler direct, parvient (en-dehors de toute considération de validité logique ou d'éthique) à utiliser de façon plus efficace que son adversaire l'arme de l'argument ad hominem. Il montre aussi comment le maniement combiné de l'attaque personnelle directe (visant un défaut de l'individu) et de l'ad hominem dit circonstanciel (visant une contradiction dans ses discours et/ou entre son discours et son comportement) permet à la fois de discréditer le concurrent et de construire une image de soi efficace en assurant la promotion de sa propre personne.

$16 \mathrm{Au}$ terme de ce parcours, je voudrais esquisser deux lignes de réflexion supplémentaires. La première touche au défi que Leff tente de relever pour répondre aux critiques des contemporains à l'égard d'une rhétorique classique considérée comme surannée. Il aborde dans ce cadre le poids des contraintes sociales et des pesanteurs institutionnelles, le rôle du pouvoir dans son rapport au discours, la question de l' «agentivité » ou capacité du sujet parlant à agir, c'est-à-dire à user de la 
parole pour exercer une action et assumer des responsabilités dans la sphère publique. La seconde ligne de réflexion concerne les difficultés dans lesquelles le rhétoricien se débat pour surmonter le fossé qui se creuse entre la théorie qui relève du général, et l'analyse du discours oratoire qui est pour lui de l'ordre du singulier.

En ce qui concerne le premier point, Leff propose une solution à ce qu'il reconnaît être l'un des problèmes centraux de la rhétorique classique, en l'occurrence l'importance primordiale accordée aux intentions et aux choix d'un orateur en pleine maîtrise de ses moyens. Il prend en compte les critiques qui recoupent ce qui a été traité en France à partir de la fin des années 1960 en termes de problématique du sujet - il s'agit globalement de rejeter des conceptions individualistes et rationalistes jugées périmées sur la base des théories de l'inconscient, de l'idéologie ou du pouvoir. Sans adopter les approches postmodernes d'une partie de ses collègues nourris de "French Theory", Leff insiste sur le déplacement qu'il importe de faire subir à la rhétorique classique en se focalisant sur le texte plutôt que sur l'orateur. Ce déplacement complexifie la notion d'intention : elle n'est plus à rechercher dans la claire conscience du sujet parlant, mais ressort de l'examen attentif du texte même (1992: 224). L'intentionnalité relève donc du discours et non des dispositions de l'orateur.

En même temps, la microlecture de Leff peut d'autant moins faire l'économie d'un sujet qui prend la parole en connaissance de cause et dans un objectif concerté, que la notion d'agentivité est au cœur du projet rhétorique ${ }^{4}$. L'agentivité (agency) pose que la parole est action et que l'orateur est un individu qui utilise délibérément les possibilités du discours pour exercer une influence sur l'auditoire et intervenir dans le réel. Comment dès lors combler le fossé entre une vision rhétorique (et pragmatique) du discours et la vision contemporaine des relations de pouvoir et des déterminations institutionnelles qui pèsent sur l'individu ? La façon dont Leff traite de la question dans son article sur l'agentivité et la tradition (traduit ici-même) est symptomatique de ce qui le rapproche et l'éloigne tout à la fois des travaux de langue française qui se réclament de l'analyse du discours et de l'argumentation rhétorique.

La particularité de la démonstration de Leff est qu'elle se fonde sur la rhétorique antique, et tout particulièrement sur celle d'Isocrate, pour résoudre une apparente incompatibilité dans la pensée contemporaine. A travers ce parcours singulier, c'est l'érudition et la compréhension en profondeur de la pensée classique qui mène à la solution d'un problème que les études rhétoriques se doivent aujourd'hui d'affronter. Il s'agit en fait de concilier l'ancrage social du sujet et sa marge de liberté, les déterminations sociopolitiques et institutionnelles du discours et son pouvoir d'invention. Selon Leff, la rhétorique classique bien comprise nous enseigne que le sujet parlant est tributaire de la culture dans laquelle il évolue; la tradition qu'il partage avec son auditoire est constitutive de son identité individuelle. Dès lors, c'est seulement à partir de cette tradition commune, qui le modèle et qui le lie à son public, qu'il peut tenter d'agir sur le réel par sa parole. En d'autres termes, les choix de l'orateur ne peuvent être coupés du discours ambiant et des opinions en cours, qui le contraignent mais qu'il tente en retour d'infléchir. La novation et l'invention sont ainsi inséparables de la tradition. Il y a donc bien "agentivité ", au sens de capacité du locuteur à agir dans la sphère publique et à y exercer une influence dont il est comptable ; mais elle est tributaire de la culture dans laquelle s'enracine l'échange verbal. Leff propose ici une approche de l'agentivité qui n'est pas sans rapport avec celle qu'avancent les études de langue française soucieuses d'articuler l'analyse du discours (où la parole du sujet est 
dépendante d'une situation de discours et d'un interdiscours), à l'argumentation rhétorique (qui insiste sur la capacité de choix et d'action, et donc la responsabilité, du locuteur) (Amossy 2009).

C'est dans ce cadre que s'est posée à Leff une question qui l'avait peu préoccupé à ses débuts, celle de l'intertextualité. Cette notion désigne en fait chez lui celle d'interdiscours située au cœur de l'analyse du discours à la française - l'ensemble des discours passés et présents qui circulent dans un état de société donné et au sein duquel se dégagent les textes avec lesquels le discours nouveau dialogue plus particulièrement. Là aussi, le parcours rappelle la situation dans laquelle s'est trouvée l'analyse textuelle (et en particulier des textes littéraires) en France lorsqu'elle a tenté d'échapper à la clôture de l'œuvre en la replongeant dans l'espace intertextuel au sein duquel elle s'élabore. Faisant retour sur son travail, Leff reconnaît que son étude de la dynamique interne du texte, qui étudie de l'intérieur sa visée d'influence, tend à l'arracher à son intertexte et de ce fait à l'isoler des controverses qui nourrissent le texte et qu'il alimente en retour (1992: 227). Le tenant de la microlecture dit chercher une voie pour replonger le discours oratoire dans l'interdiscours de l'époque sans pour autant dissoudre l'analyse textuelle dans l'étude globale d'une formation discursive, des rapports de pouvoir, d'une idéologie. Dans un retour réflexif sur ses études anciennes du discours de Cooper Union datées du début des années 1970, Leff propose en 2001 de les actualiser en tenant compte de l'intertexte. On ne peut isoler le texte, et même son rattachement à un genre de discours (ici, le discours électoral) ne suffit pas : il doit être mis en relation avec les motifs qui construisent la polémique sur l'esclavage et avec les interprétations contradictoires que les adversaires font de la Constitution et de la Déclaration d'Indépendance américaine (4 juillet 1776); il doit également être compris dans son rapport au débat de Lincoln avec son rival immédiat, Douglas. Il n'en reste pas moins que ces éléments intertextuels doivent être analysés dans le texte même: ce sont les modalités au gré desquelles celui-ci les intègre dans sa structure, et la façon dont ils contribuent à modeler sa visée rhétorique, qu'il faut décrire. En termes bakhtiniens, on dirait qu'il s'agit d'explorer la dimension dialogique et dialogale des textes. Il est intéressant de noter que dans le texte théorique de 1992, "Things made by Words", Leff propose, comme objet d'étude privilégié pour une analyse textuelle en prise sur l'intertexte, l'étude des controverses qui ne sont pas, selon lui, une formation discursive mais un ensemble de discours particuliers pris dans un jeu d'oppositions où il est possible, au-delà des points récurrents, de s'arrêter sur certains des textes en situation.

21 Mettant en avant la prépondérance du discours oratoire concret et d'une pratique analytique attachée à la singularité de son objet, Leff reconnaît que cette approche n'est pas sans entraver la possibilité de dégager des règles générales et de décrire des fonctionnements discursifs communs. Au gré de la démarche réflexive qui lui est propre, il essaye de voir comment il est possible de gérer la tension entre le singulier et le général :

Cette approche s'attache à comprendre les discours dans toute leur complexité en les appréhendant à la fois comme des constructions linguistiques et comme des tentatives d'exercer une influence, et elle opère à l'aide de cas paradigmatiques plutôt que de principes abstraits [...] Avec le temps, le critique développe un répertoire de textes de ce type et une sensibilité accrue aux possibilités de la pratique rhétorique issue de l'étude concrète de textes en situation. De cette façon, le critique s'efforce de cultiver la capacité, le jugement pratique nécessaire à l'interprétation et à l'évaluation de tout cas nouveau qui se présente et le jugement 
se déplace latéralement à travers des cas de figure, fixant ainsi l'attention sur des performances rhétoriques matérialisées plutôt que sur des formes abstraites (Leff $1992: 228$, je traduis). $d$ analyse et en l'absence d'outils notionnels. Si les dispositifs et les taxinomies hérités de la tradition rhétorique sont ici instrumentaux (Leff ne se tourne guère vers les sciences du langage, qui ne font pas partie de sa formation d'analyste), ces outils ne doivent jamais faire l'objet d'une application mécanique. Ils sont mobilisés pour dégager la spécificité du texte et parvenir à une compréhension adéquate de ce qui s'y trame. Ce va-et-vient entre l'analyse concrète et les régulations abstraites, entre l'appréhension d'une singularité et la mise au jour de fonctionnements communs, marque sans doute chez le rhétoricien une tension non résolue. Or celle-ci - et c'est là un point important - ne lui appartient pas en propre. Les difficultés auxquelles le tenant de la microlecture se mesure dévoilent le dilemme dans lequel sont souvent pris les analystes soucieux à la fois de dégager certaines régulations, et de saisir le particulier sans le réduire.

On aura noté au passage que la microlecture examine la construction du discours politique pour mieux en déchiffrer le sens et en dégager les enjeux. Dans cette perspective, elle est effort de compréhension et d'interprétation; elle se réclame d'une herméneutique. Et en effet, Leff parle à maintes reprises d'activité interprétative et dans son article sur « La rhétorique herméneutique » (1997), il s'oppose à l'idée avancée par certains rhétoriciens américains que la rhétorique classique et contemporaine se distinguent l'une de l'autre en ce que la première s'attache au langage comme pouvoir et se veut initiation à la pratique oratoire, tandis que la seconde s'attache au langage comme signification et voit dans le texte l'espace de la production de sens (1997: 195). Se tournant vers la tradition classique en l'éclairant, à son ordinaire, d'un jour nouveau, Leff montre comment la pratique de l'imitatio dément la thèse d'une coupure entre la production et l'interprétation des discours. L'imitatio demande en effet d'examiner un modèle pour «en identifier les stratégies et les formes telles qu'elles sont concrétisées dans un texte " historiquement situé, et de dégager la signification qui se construit dans l'ensemble du texte en relation avec ses objectifs rhétoriques particuliers (ibid. : 202). En un deuxième temps, l'analyse et l'interprétation du discours passé doivent servir à la production d'un discours nouveau en tenant compte de sa situation particulière. Cette rhétorique herméneutique fondée sur l'imitatio, c'est ce que pratiquent les grands orateurs - Leff reprend dans son article un exemple tiré de Lincoln. Mais si l'on se reporte à l'analyse rhétorique, qui n'est pas en soi un exercice de pratique oratoire, on peut voir qu'elle est autorisée à reprendre à son compte la démarche interprétative qui est, selon Leff, inhérente à la rhétorique classique.

Il me semble intéressant de souligner que la critique rhétorique du discours politique se rapproche paradoxalement ici de l'analyse littéraire. En effet, elle entend explorer un texte dans son unicité et sa complexité propre. Elle utilise pour ce faire des instruments tirés de la rhétorique classique, mais fait aussi preuve de l'attention soutenue aux structures et aux formes verbales (construction du texte, indications temporelles, rythme, métaphores, figures de style, etc.) qui caractérise les études 
littéraires. Elle lie étroitement la construction formelle et la production du sens pour effectuer un déchiffrement qui se donne comme un travail d'interprétation. Qui plus est, Leff sélectionne les grands discours politiques - ceux qui, dans leur densité et leur complexité, lui paraissent exemplaires et peuvent être qualifiés de véritable art oratoire. Enfin, on a vu que pour rendre compte de ces remarquables usages du discours en situation, la microlecture met souvent au second plan l'investigation des constantes et l'étude des régulations globales. Dans un sens, on peut dire que Leff veut hisser l'analyse de l'allocution publique au rang du type d'analyse dont était seul jugé digne le texte littéraire - et cela, paradoxalement, lors même qu'il jette les fondements d'une analyse du discours politique considéré dans sa spécificité.

Notons enfin une dernière tension, elle aussi féconde (et sur laquelle on trouvera un commentaire intéressant dans l'article de Jasinski sur le close reading [2001]). Les travaux de Leff, qui se réclament d'une pratique concrète de l'analyse, sont en fait toujours soucieux de sonder une question théorique. Le rhétoricien se dit voué à une pratique analytique des discours dans leur matérialité concrète, qui l'écarte des constructions théoriques abstraites. En même temps, l'étude des textes particuliers lui donne l'occasion d'examiner un problème, ou de mettre à l'épreuve une notion : tantôt c'est la question des genres, tantôt celle de l'argument ad hominem dans son rapport à l' ethos, ou celle de la divergence entre la logique informelle et la rhétorique, ou encore celle de l'intertextualité et de son intégration dans la microlecture. Souvent les études concrètes sont précédées d'un questionnement théorique et méthodologique qui dirige la lecture ; si c'est le questionnement qui est mis en avant, il est mis à l'épreuve sur un exemple concret. Ce va-et-vient entre l'exploration d'une question théorique, qui porte sur le général, et la pratique des textes, qui explore le particulier, est symptomatique d'une approche rhétorique qui se veut enracinée dans la matérialité des discours et qui est néanmoins désireuse de s'interroger sur le fonctionnement global du discours public à visée persuasive.

Sans doute ai-je été tentée, en présentant les travaux de Michel Leff et leurs rapports aux disciplines connexes (analyse du discours, argumentation, études littéraires) en territoire francophone, à utiliser les formulations qui leur sont propres. Si cette «traduction » marque plus fortement que la terminologie utilisée par le rhétoricien américain la proximité des questionnements, des objectifs et des démarches, elle ne doit pas dissimuler que les recherches ne se confondent pas. Il ne s'agit pas ici d'assimiler l'une à l'autre des approches différenciées, mais de souligner l'actualité et l'apport d'un travail profondément ancré dans la rhétorique classique, qui cherche à frayer des voies nouvelles à une analyse rhétorique résolument contemporaine centrée sur le discours politique et l'examen minutieux des grands textes de l'art oratoire américain. 


\section{BIBLIOGRAPHIE}

Amossy, Ruth. 2002. «L'argument ad hominem dans l'échange polémique », Declercq, Gilles, Michel Murat \& Jacqueline Dangel (éds). La parole polémique (Paris : Champion), 409-423

Amossy, Ruth. 2009. « Argumentation in Discourse : A Socio-discursive Approach to arguments », Informal logic 29, 252-267

Amossy, Ruth. 2010 [2000]. L'argumentation dans le discours (Paris : Colin)

Amossy, Ruth. 2010b. La présentation de soi. Ethos et identité verbale (Paris : PUF)

Brinton, Alan. 1985. « A Rhetorical View of the ad hominem », Australasian Journal of Philosophy 63, 51-63

Charaudeau, Patrick \& Dominique (éds). 2002. Dictionnaire d'analyse du discours (Paris : Seuil)

Charaudeau, Patrick. 2008. "L'argumentation dans une problématique d'influence ", Argumentation et Analyse du Discours 1, http://aad.revues.org/index193.html [consulté le 24.3.2011]

Geisler, Cheryl. 2005. « Teaching the Post-Modern Rhetor. Continuing the Conversation on Rhetorical Agency », Rhetorical Society Quarterly 34, 107-113

Jasinski, James. 2001. « Close reading ", Sourcebook on Rhetoric. Key Concepts in Contemporary Rhetorical Studies (Thousands Oak/London/New Delhi : Sage Publications), 91-97

Leff, Michael. 1986. "Textual Criticism : The Legacy of G. P. Mohrmann », Southern Journal of Speech Communication 51, 337-389

Leff, Michael. 1992. «Things Made by Words : Reflections on Textual Criticism », Quarterly Journal of Speech 78, 223-231

Leff, Michael. 1997. « Hermeneutical Rhetoric », Jost, Walter \& Michael Hyde (eds). Rhetoric and Hermeneutics in our Time (New Haven: Yale University Press), 196-214

Leff, Michael. 2001. «Lincoln at Cooper Union : Neo-Classicism Revisited », Western Journal of Communication $65,323-348$

Leff, Michal \& Ebony Utley. 2004. «Instrumental and Constitutive Rhetoric in Martin Luther King's 'Letter From a Birmingham Jail' », Rhetoric and Public Affairs 7, 37-52

Leff, Michael. 2009. « Perelman, ad Hominem Argument, and Rhetorical Ethos », Argumentation 23, 301-311. Version française : 2009. " Perelman, argument ad hominem et ethos rhétorique », Argumentation et Analyse du Discours 2

Maingueneau, Dominique. 1997 [1991]. L'Analyse du Discours (Paris : Hachette)

Maingueneau, Dominique. 2004. Le discours littéraire. Paratopie et scène d'énonciation (Paris : Colin)

Perelman, Chaim \& Lucie Olbrechts Tyteca 1970 [1958]. Traité de l'argumentation. La nouvelle rhétorique (Bruxelles : Éditions de l'Université de Bruxelles)

Rhetoric and Public Affairs $13: 4,2010$, «Celebrating the Life of Michael Leff » 


\section{NOTES}

1. On trouvera ailleurs des présentations intéressantes du travail de Michael Leff dans la perspective de la rhétorique américaine et en particulier dans Rhetoric and Public Affairs 13:4, 2010.

2. C'est précisément à cause de l'allégeance de l'argumentation dans le discours aux sciences du langage, et de sa capacité à apprehender les textes de manière fine grace aux notions issues de diverses branches de la linguistique, que Michael Leff m'avait demandé de participer au séminaire de Critique rhétorique qu'il avait organisé à l'Institut d'été de la RSA en 2009.

3. Selon Maingueneau (1997 [1991] : 13), l'analyse du discours ne se réduit «ni à l'analyse linguistique d'un texte ni à une analyse sociologique ou psychologique du 'contexte' » : elle " doit penser le dispositif d'énonciation qui lie une organisation textuelle et un lieu social déterminé ». 4. Voir à ce propos Geisler 2005.

\section{AUTEUR}

RUTH AMOSSY

Université de Tel-Aviv, ADARR 